\title{
La personalización educativa en tiempos de cambio e innovación educativa. Un ejemplo ilustrativo
}

\section{Moisés Esteban-Guitart}

Universitat de Girona, Girona, España

mail: moises.estebandudg.edu

ORCID: https://orcid.org/0000-0002-1700-8792

\section{Edgar Iglesias}

Universitat de Girona, Girona, España

mail: edgar.iglesiasvaudg.edu

ORCID: https://orcid.org/0000-0002-0356-0439

\author{
Javier González-Patiño \\ Universidad Autónoma de Madrid, Madrid, España \\ mail: javier.qonzalezpatinoQuam.es \\ ORCID: https://orcid.org/0000-0003-3909-1897 \\ Irene González-Ceballos \\ Universitat de Girona, Girona, España \\ mail: Irene97gonzalezQिgmail.com
}

\section{RESUMEN}

En este artículo se describe e ilustra la personalización educativa entendida como un conjunto diverso de recursos, estrategias y actuaciones encaminadas a facilitar que los aprendices otorguen sentido y valor personal a los aprendizajes. Se proponen y consideran seis principios o elementos de la personalización así entendida, a saber: iniciar con el reconocimiento de las identidades de los aprendices, estimular procesos de reflexión, fomentar la agencia del aprendiz, promover trayectorias de aprendizaje, establecer procesos de vinculación-conexión múltiple o bimodal y facilitar mecanismos y procesos de acompañamiento socio-educativo. Tras una descripción de los mismos, se ilustran a través del proyecto "¡Cápsulas! Itinerarios de aprendizaje" llevado a cabo en un instituto de secundaria de titularidad pública en el tercer semestre del curso académico 2019-2020, coincidiendo en el período de confinamiento debido a la pandemia Covid-19. Se concluye considerando lo que nos parecen tres retos, también oportunidades, de la enseñanza remota o educación virtual.

Palabras clave: aprendizaje, personalización educativa, cambio e innovación educativa, trayectorias de aprendizaje.

\section{Personalized education during a period of educational change and innovation. An illustrative example}

\section{ABSTRACT}

This article examines and empirically illustrate the personalized education conceived as a sum of different strategies, actuations and resources to promotes learners' sense-making in the teaching and learning process. Six principles or elements involved in personalized education are suggested: starting from recognizing students' identities, fostering reflexive practices, facilitating the learner's agency, developing learning pathways, promoting the multiple linking-bridging, and implementing socio-educative supports and scaffolds. After describing those principles, the project "¡Cápsulas! Itinerarios de aprendizaje” allow us to illustrate the elements previously described. This project was designed and implemented during the third semester of the course 2019-2020, within confinement circumstances caused by Covid-19 pandemic. It is concluded by considering three challenges and opportunities of virtual education or remote teaching from the empirical example examined here.

Keywords: learning, personalized education, educational change and innovation, learning pathways. 


\section{Contextualización}

Las instituciones de educación formal (escuelas, institutos, universidades), con una intención explícita, deliberada y sistemática concretada en un currículo oficial, coexisten con una tensión intrínseca entre la herencia de su pasado y las circunstancias presentes-futuras a las que deben responder. En este sentido, distintos autores han puesto de manifiesto la necesidad de (re) pensar las instituciones educativas públicas para que no pierdan su sentido o razón de ser (Álvarez-Arregui y Arreguit, 2019; Coll, 2009, 2014; Autores, 2014; Tarabini, 2020; Tedesco, 2001). Idea que cobra, si cabe, un nuevo protagonismo a raíz de las consecuencias derivadas de la pandemia de la Covid-19, lo que se ha dado en llamar "educación post-pandemia" (Iglesias, González-Patiño, Lalueza y Esteban-Guitart, 2020; Pardo y Cobo, 2020).

Se estima que más de mil millones de estudiantes y jóvenes, lo que supone un porcentaje superior al 70\% del total de alumnos/as matriculados, de unos 190 países en todo el mundo, se vieron afectados por el cierre de escuelas y universidades debido al brote de la Covid-19, identificada por vez primera durante el mes de diciembre del año 2019 en Wuhan, capital de la provincia de Hubei, en la República Popular de la China ${ }^{1}$. En el caso del contexto español, el confinamiento de los y las estudiantes en sus hogares, con el cierre de los centros educativos, se inició el 13 de marzo de 2020. Ello obligó a realizar el último semestre y la finalización del curso académico 2019-2020 de forma no presencial. Afectaciones que se prolongaron en el siguiente curso 2020-2021.

Derivado de esta situación excepcional, se han sugerido distintos recursos, metodologías y estrategias para reforzar los procesos de educación remota, a distancia u online. Por ejemplo, la Sociedad Internacional de Tecnología en Educación (ISTE) supervisó un amplio abanico de recursos de libre acceso, organizados por niveles, categorías y lenguas ${ }^{2}$. Paralelamente, se sugirieron guías para dar una respuesta educativa al nuevo escenario (ver, por ejemplo, Reimers y Schleicher, 2020).

No obstante, más que recursos y soluciones concretas de auxilio, nos parece relevante situar la actuación educativa en una comprensión o planteamientos pedagógicos que permitan no solamente justificarla sino sobre todo orientarla para, principalmente, dar sentido a la práctica educativa realizada. En este sentido, estamos de acuerdo con la propuesta de educación híbrida de autores como Pardo y Cobo (2020), quienes abogan por superar las barreras arquitectónicas tradicionales entre la formación presencial y virtual. En este sentido, se invita a transitar hacia experiencias de aprendizaje expandidas, líquidas y distribuidas en una línea de tiempo narrativa multimedia a través de la cual los docentes y estudiantes puedan servirse de modo colaborativo de lo mejor de la presencialidad y la cultura digital.

El objetivo principal de este artículo consiste en ilustrar la pertinencia de la personalización educativa, en general, así como en los escenarios de educación post-pandemia se refiere, en particular. Para ello, dividimos el texto en tres secciones. En primer lugar, justificamos los que nos parecen los elementos centrales de la personalización, en el ámbito educativo. En segundo lugar, ilustramos su actualización en tiempos de pandemia a través de un proyecto educativo llevado a cabo en un instituto de reciente creación en Sabadell, Catalunya. Dicho análisis de caso nos permite identificar y ejemplificar los principios y elementos centra-

1 Se puede consultar la actualización de los datos (según UNESCO), así como la Coalición Mundial para la Educación, a través del siguiente enlace: https://es.unesco.org/covid19

2 Accesible a través de https://www.learningkeepsgoing.org/freetech-for-learning les de la personalización educativa. Finalmente, a modo de conclusión, consideramos algunos riesgos, también oportunidades, a propósito del ejemplo descrito.

\section{Propósito y principios de la personalización educativa}

Entendemos por personalización educativa un conjunto diverso y heterogéneo de estrategias, recursos y actuaciones pedagógicas encaminadas a promover aprendizajes con sentido y valor personal por parte de los y las estudiantes (Coll, Esteban-Guitart e Iglesias, 2020). Una primera aclaración, por tanto, nos lleva a utilizar el concepto de personalización educativa, en lugar de nociones más utilizadas en la literatura como son las de personalización del proceso de aprendizaje o aprendizaje personalizado -personalized learning en inglés (Chen, 2000; Grant y Basye, 2014; Martinez, 2001; Minguillón, Mor, Santanach y Guàrdia, 2005). Con ello queremos enfatizar que la personalización es un medio, entre otros, para promover un fin, a saber: que el aprendiz pueda dar sentido y valor a las distintas experiencias de aprendizaje. Es decir, mientras que la personalización comprende distintas actuaciones educativas, en un nivel social y material ("interpsicológico-social"), el aprendizaje se refiere a la potencial consecuencia de las mismas, siendo un proceso de construcción/ apropiación de conocimientos y/o desarrollo de competencias ("intrapsicológico-personal") (Autores, 2020; Vygotsky, 1984).

Otra aclaración preliminar, vinculada a la anterior, nos lleva a considerar la personalización como un conjunto de ayudas, sociales y materiales, lejos de suponer un proceso individual e individualizado de adquisición y construcción de conocimientos (Penuel y Johnson, 2016), así como una mera "costumización" del proceso de aprendizaje (Pérez Guerrero y Ahedo Ruiz, 2020). Más en particular, Coll, Esteban-Guitart e Iglesias, 2020 definen la personalización como:

“Un conjunto de propuestas, estrategias, recursos y actuaciones encaminadas a conectar los aprendizajes con los intereses, decisiones, proyectos y experiencias del aprendiz, siendo la finalidad última que el aprendiz pueda dar sentido y valor personal a lo que aprende (...) Es aquella actividad, práctica o experiencia que vincula los intereses, objetivos y opciones de los aprendices con los objetivos y actividades educativas a partir de su participación-implicación en el control-elección de los procesos implicados (qué, cómo y cuándo aprender)" (pp. 9-35).

En este sentido, un aprendizaje con sentido y valor personal se caracteriza por experiencias subjetivas de aprendizaje, ya sean originadas en el ámbito formal o informal, que, por su impacto emocional y cognitivo, permiten al aprendiz conocer más y mejor su historia personal y la realidad que le rodea, ofreciendo mejores condiciones para actuar en ella, así como le permiten crear planes de actuación futura (Coll, 2018). Es decir, un aprendizaje tiene valor y sentido personal cuando ayuda al alumnado a revisar, reconstruir y entender experiencias pasadas, situaciones presentes y a proyectar su futuro, en el plano personal, cívico y social, o académico y profesional.

En la Figura 1 presentamos seis dimensiones, elementos o componentes de un mismo proceso interrelacionado que gira alrededor de la personalización educativa. Dichas dimensiones no excluyen, ni pretenden reducir, el proceso de personalización educativa, sino más bien analizarlo con el fin de comprenderlo más y mejor. 


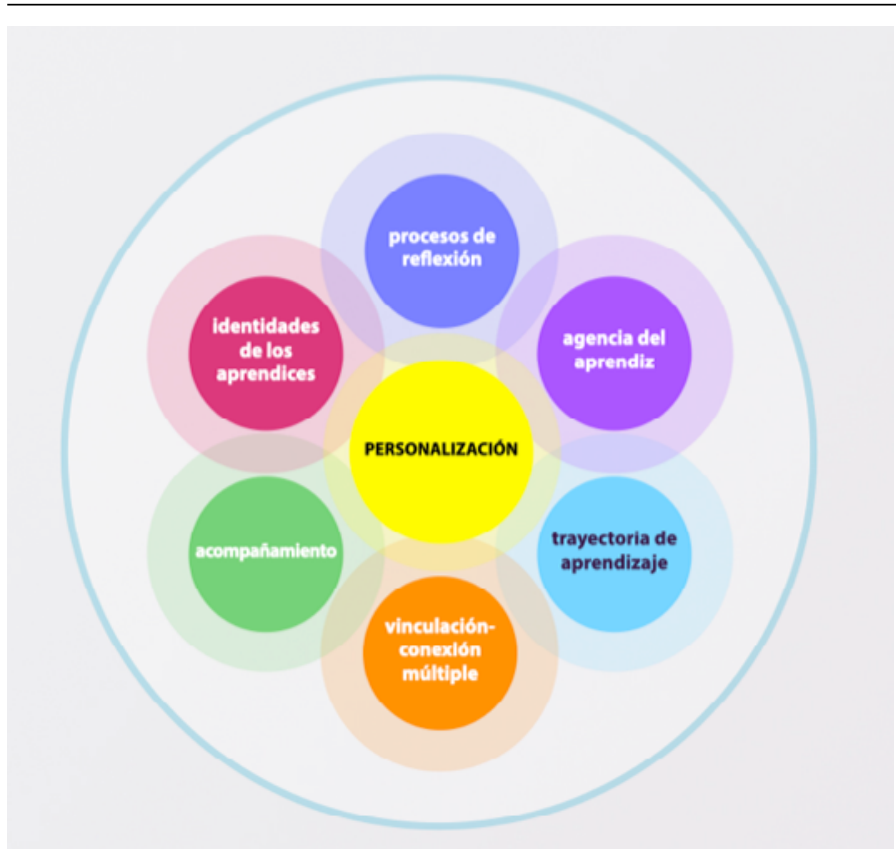

Figura 1. Elementos de la personalización educativa. Fuente: Elaboración propia

En primer lugar, la actuación pedagógica debe partir de las identidades en curso de los y las estudiantes. Ello supone identificar aquellos elementos que son percibidos como relevantes, significativos, por parte de los aprendices, pudiendo ser estos: intereses, conocimientos previos, personas, actividades, instituciones, o artefactos culturales. Supone también atender a la dimensión proyectiva de la identidad en tanto que decisiones y proyectos de vida. En este sentido, entendemos por identidad un proceso constante de (re)significación a partir del impacto y valoración de las personas, actividades, objetos, sitios e instituciones percibidas como significativas por parte del aprendiz en cuestión (Esteban-Guitart, 2016, 2019).

Preferimos la noción de "identidad" frente a la de "interés", comúnmente asociada a la personalización del aprendizaje (Walkington y Hayata, 2017), pues la primera engloba la segunda, siendo más expansiva. Por otra parte, más allá de las clásicas conceptualizaciones, de raíz cognitiva, alrededor de la identidad en tanto que auto-concepto académico (Moronta, Rodríguez-Fernández y Fernández-Lasarte, 2019), vinculamos la identidad al ámbito subjetivo del sentido del aprendiz, es decir, aquellos objetos, decisiones, proyectos, conocimientos, creencias, personas, actividades o espacios que son reconocidos como especialmente importantes y relevantes para el aprendiz (Esteban-Guitart, 2016; Esteban-Guitart y Moll, 2014; Subero y Esteban-Guitart, 2020). Los intereses, en cambio, pueden reducirse a aspectos más superficiales como determinadas preferencias o gustos personales (González-Patiño y Esteban-Guitart, 2019).

Cabe aclarar, por otra parte, que lejos de considerarse un producto final de la situación educativa, las identidades se conceptualizan como el inicio del proceso pedagógico en constante transformación a través de procesos de andamiaje. Es decir, a través de la vinculación de un elemento identitario en particular con un contenido, competencia u objeto de aprendizaje, las mismas identidades se van configurando y desarrollando (Esteban-Guitart, 2019). Para dicho fin, se han sugerido los "artefactos identitarios" en tanto que producciones realizadas por el propio aprendiz a través de las cuales proyectan sus intereses, aficiones, actividades, personas, objetos y espacios percibidos como especialmente significativos, y utilizados por los y las docentes con finalidades curriculares y pedagógicas (Autores, 2019; Autores,
2018). Un ejemplo de artefacto identitario es el "dibujo significativo" a través del cual el estudiante señala en el interior de un círculo los elementos más relevantes, que le caracterizan e identifican mejor. Más concretamente, con figuras redondeadas se identifican personas significativas y mediante cuadrados se identifican actividades, objetos, instituciones, lugares percibidos como significativos. Teniendo en cuenta que cuanto más en el centro del dibujo se ubican dichos elementos, más significativos son -tienen más valor- para el aprendiz (ver Figura 2).

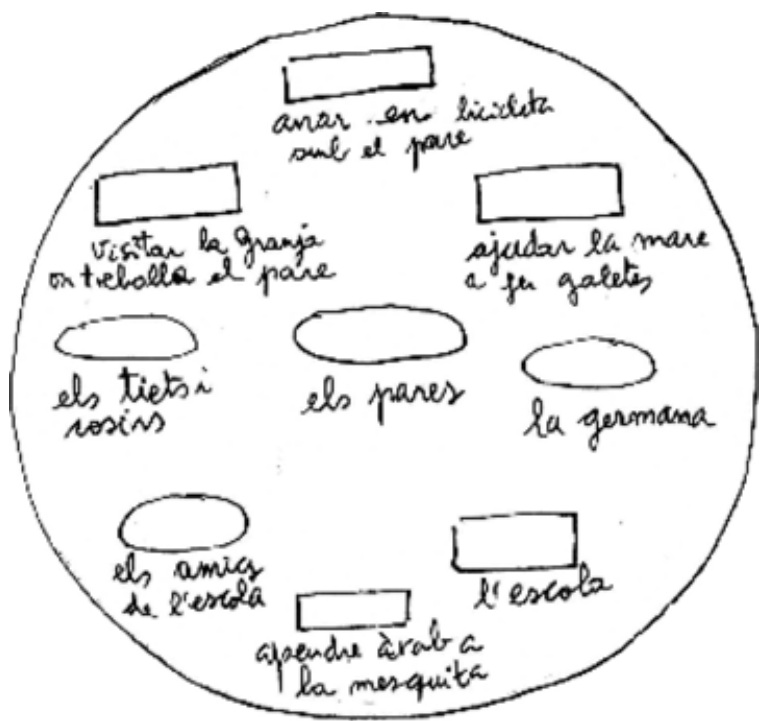

Figura 2. Ejemplo de círculo significativo. Fuente: Jovés, Siqués y Esteban-Guitart $(2015, p .75)$

En la Figura 2 se muestra un ejemplo de artefacto identitario (círculo significativo) de una alumna de 7 años de edad. En dicha representación aparecen referencias a la familia, a la escuela, al aprendizaje del árabe en la mezquita, a la realización de galletas con su madre, así como visitar la granja donde trabaja su padre. A partir precisamente de esta última referencia, el docente diseñó una unidad didáctica alrededor de animales de granja para tratar contenidos, mediante la visita a dicha granja, como aprender a clasificar los animales según lo que comen (herbívoros, carnívoros y omnívoros), descubrir los beneficios y productos obtenidos de animales de granja, o expandir vocabulario a través de un diccionario multilingüe sobre animales mediante la utilización de las lenguas maternas de los estudiantes, así como el catalán, castellano e inglés (Jovés, Siqués y Esteban-Guitart, 2015).

En este sentido, las identidades en construcción sirven como experiencia previa y significativa a partir de la cual se pueden vehicular los objetivos de aprendizaje académicos. De hecho, distintos estudios muestran que los aprendizajes más resilientes, que tienen un mayor impacto en la memoria a largo plazo, son aquellos que parten y se conectan con las necesidades, identidades, intereses y objetivos de los aprendices (Barron, 2006; Status, Falk, Penuel, Dierking, Wyld y Bailey, 2020).

Para ello se requiere de procesos de reflexión, personal y grupal, que permitan identificar dichos intereses, proyectos, identidades y/o aprendizajes, así como enriquecerlos. Por ejemplo, a través de una tabla o mapa que permita identificar-explicitar un determinado interés o proyecto identitario del estudiante, su origen y desarrollo, así como las consecuencias que se derivan del mismo, más allá de si este interés u objetivo-objeto identitario ha surgido en el contexto escolar-formal, o bien en otras situaciones y contextos. En definitiva, los intereses, identidades y procesos 
de desarrollo y aprendizaje de los estudiantes son el resultado de procesos dialógicos de discusión-reflexión (Oliveira y Coelho, 2020).

Existen distintos recursos para propiciar la identificación y reflexión sobre, por ejemplo, experiencias de aprendizaje. Desde diarios personales en los que anotar el aprendizaje más significativo realizado a lo largo del día, y toda la ecología que le rodea (con quién se ha realizado qué ha sentido o experimentado, cómo se ha dado, dónde se ha dado) ((Esteban-Guitart, Serra y Vila, 2017); hasta cartografías (mapas de aprendizaje) para explorar espacios y trayectorias particulares de aprendizaje (ver Figura 3).

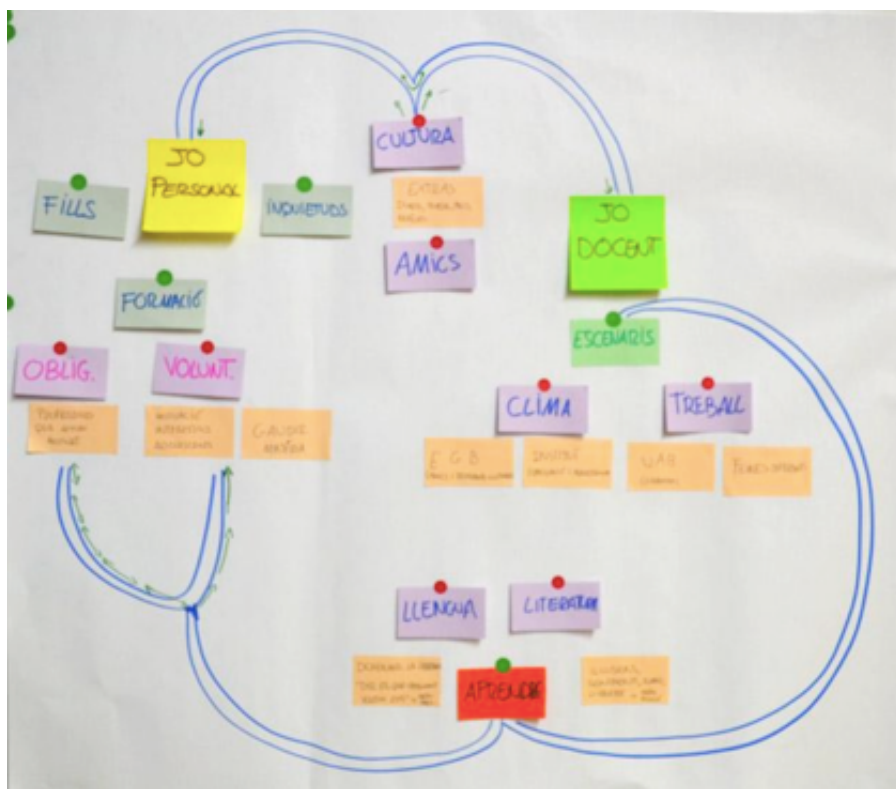

Figura 3. Ejemplo de cartografía de aprendizaje. Fuente: Hernández-Hernández, Sancho-Gil y Domingo-Coscollola (2018, p. 111)

Otra manera de trabajar el ámbito de las identidades (desde su dimensión personal de sentido) es facilitando lo que aquí llamamos "procesos de agencia". Es decir, que el aprendiz tome responsabilidad, participación y control en los procesos de aprendizaje: en el establecimiento de objetivos de aprendizaje, participación en el co-diseño de las actividades, así como evaluación de los aprendizajes alcanzados. Se ha documentado que una mayor participación de los y las estudiantes en los procesos y fases relativas al aprendizaje deviene en una mayor implicación escolar, así como un mejor rendimiento y aprovechamiento académico (Perin, 2011). Una de las maneras de propiciar estos procesos de agencia por parte del estudiante es a partir de su participación directa en el establecimiento y desarrollo de planes personales de aprendizaje que acaban definiendo itinerarios o trayectorias de aprendizaje particulares. Los contextos formales tradicionales de enseñanza y aprendizaje responden a un modelo homogéneo de escolarización universal (Coll, 2013). Sin embargo, existe una creciente visión compartida en considerar la multiplicidad de escenarios, agentes, servicios y oportunidades de aprendizaje que trascienden hoy los espacios y tiempos educativos escolares (Álvarez-Arregui y Arreguit, 2019; Barron, 2006; Autores, 2018; Nasir, de Royston, Barron, Bell, Pea, Stevens y Goldman, 2020; Autores, 2017).

Tal y como ha evidenciado la reciente situación derivada de la Covid-19, aparecen un sinfín de posibilidades en el ámbito informal y a través de los medios digitales y la web social no solo en el acceso a la información, o contenidos específicos, sino también en la creación de conocimientos, experiencias, productos culturales y la difusión de los/as mismos/as en comunidades y redes de afinidad (Gee y Esteban-Guitart, 2019; González-Patiño y Esteban-Guitart, 2014).

Sin embargo, que el aprendizaje se dé hoy necesariamente a lo largo y a lo ancho de la vida (Esteban-Guitart, Coll y Penuel, 2018), no significa necesariamente que las experiencias de aprendizaje estén conectadas; ni que lo estén los contextos o actividades que en ellos/ellas se realizan. Para este fin, es necesario actuaciones de vinculación-conexión explícita en dichos niveles: mediante actuaciones que faciliten las conexiones entre las experiencias de aprendizaje de los estudiantes -a nivel psicológico-individual-, así como que permitan conectar agentes, tiempos y espacios educativos, por ejemplo centros educativos, familias y entidades comunitarias (Rodríguez-Ruiz, Álvarez-Blanco, Martínez-González y Epstein, 2019). Por eso hablamos de vinculación-conexión múltiple o bimodal (ver Figura 1).

Dicho con otras palabras, la vinculación es doble, por una parte, a nivel micro, se interrelacionan experiencias de aprendizaje: se conectan conocimientos y experiencias previas de aprendizaje con nuevos conocimientos y nuevas experiencias ("vinculación psicológica"). Por otra parte, a nivel meso, se establecen lo que Bronfenbrenner (1985) consideraba relaciones de "continuidad educativa" entre distintos contextos de vida y socialización que, en definitiva, permiten mejorar su potencial evolutivo, de aprendizaje, de cada uno de estos nichos o contextos de vida y actividad ("vinculación comunitaria"). Más específicamente y, en el marco de su noción de "mesosistema", el autor consideraba que:

El potencial de desarrollo de un escenario de crianza se ve incrementado en función del número de vínculos sustentadores entre ese escenario y otros contextos en los que se insertan el niño y los adultos responsables de su cuidado. Tales interrelaciones pueden adoptar la forma de actividades compartidas, comunicación en los dos sentidos e información suministrada a cada escenario sobre los demás (Bronfenbrenner, 1985, pp. 51-52).

En otro texto, el autor expresaba la misma idea con estas palabras: "El potencial evolutivo de los entornos de un mesosistema se ve incrementado si los roles, actividades y díadas en las que participa la persona vinculante con otros entornos estimula la aparición de confianza mutua, una orientación positiva, el consenso de metas entre entornos, y un equilibrio de poderes progresivo que responda a la acción en nombre de la persona en desarrollo" (Bronfenbrenner, 1987, p. 58).

Finalmente, se han identificado condiciones tanto materiales, como sociales, para que dichas conexiones puedan darse, así como subyacen a trayectorias personalizadas de aprendizaje movilizadas por determinados objetivos e intereses por parte del aprendiz (DiGiacomo, Van Horne, Van Steenis y Penuel, 2018). Es en este sentido en el que hablamos de acompañamiento, tanto como proceso de reconocimiento y ayuda pedagógica-social, como condiciones materiales que posibiliten la vinculación de las identidades, proyectos y objetivos de los aprendices con las oportunidades, servicios, agentes y recursos, ya sean online $u$ offline, de su alrededor.

A pesar de que el modelo teórico de la personalización educativa sintetizado en la Figura 1 se ha concebido para dar cuenta de procesos de aprendizaje en situaciones de "normalidad" social, la reciente pandemia Covid-19 supone una situación educativa extraordinaria que a nuestro criterio refuerza procesos de personalización educativa, al estar imposibilitadas las condiciones y opciones de presencialidad física y escolar convenciona- 
les. En este sentido, presentamos a continuación un ejemplo que apoya dicha tesis y que permite ilustrar empíricamente, por otra parte, el funcionamiento y articulación de los principios anteriormente descritos. Se trata de un proyecto de cambio e innovación educativa basada en la personalización educativa.

“¿Cápsulas! Itinerarios de aprendizaje”. Una práctica educativa personalizada en situación de confinamiento

La experiencia “ ¡Cápsulas! Itinerarios de aprendizaje” se realizó en un instituto de titularidad pública del municipio de Sabadell de la provincia de Barcelona, en particular el Instituto Ca n'Oriac, en el contexto del tercer semestre del curso académico 2019-2020 condicionado por el cierre de los centros educativos y la cancelación de los procesos físicos de enseñanza y aprendizaje.

Se trata de un instituto de secundaria de reciente creación que contaba en dicho curso con alumnos y alumnas de primero y segundo de la ESO, por lo tanto, estudiantes de entre 12 y 14 años de edad. El centro educativo está situado en un barrio periférico del municipio con un elevado índice de diversidad sociocultural y económica.

El proyecto "¡Cápsulas! Itinerarios de aprendizaje” (ver Figura 4) se estructura en torno a los siguientes quince temas o cápsulas de aprendizaje: "body percussion", "oh la la la France!", "scratch", "PoemART", "autorretratos para el recuerdo", "ecogames", "viajemos por la historia", "once upon a time", "vacaciones solidarias", "la magia del mar", "gafas científicas", "natura urbana", "geometría doméstica", "rap" y "la naturaleza de la luz." Se trata de actividades que contemplan globalmente, y transversalmente, varios contenidos y competencias de los cursos primero y segundo de la ESO.
Cada cápsula se explica y desarrolla a partir de siete puntos ${ }^{3}$ que contemplan las instrucciones y procedimiento de las distintas actividades. En primer lugar, se responde a la pregunta "¿en qué consiste esta cápsula?", seguido de indicaciones en relación con el procedimiento (por ejemplo, dónde y cómo subir las tareas o directrices relativas a la evaluación). Finalmente, se ofrecen materiales de apoyo, así como se sugiere el itinerario específico de trabajo en las distintas tareas.

Por ejemplo, la cápsula "gafas científicas" invitaba a los y las estudiantes a observar su alrededor, sus respectivas vidas cotidianas, con el fin de realizarse preguntas sobre determinados fenómenos que puedan ser abordados mediante planteamientos y razonamientos científicos. El producto final consistía en un archivo de vídeo en el que se explicaba qué fenómenos se observaron y analizaron científicamente en las distintas situaciones cotidianas. Para ello, el o la estudiante debía elegir, con la ayuda de su tutor/a, qué pasos realizar para llegar al producto final, así como, a partir de distintos recursos y material bibliográfico propuesto, planificar y llevar a cabo un determinado itinerario de aprendizaje. Al igual que en otras cápsulas, se disponía de un modelo-ejemplo del producto final, así como de una descripción del proceso de entrega a partir de la utilización del correo electrónico, Google Drive, Classroom, o la aplicación Padlet.

Desde otra cápsula, "poemART", se pretendía identificar las conexiones entre el arte y la palabra a partir de un taller de creación que vinculaba poesía y artes plásticas, mediante el conocimiento de distintos artistas contemporáneos (Joan Brossa, Joseph Cornell, Kass Copeland). Específicamente, se propusieron tres tareas, a saber: 1) Pensar y traducir tres colores que le gustasen al o la aprendiz en versos poéticos (ejemplo-modelo: "Blanco es el color del plato de quién pasa hambre", Yousra); 2) Buscar

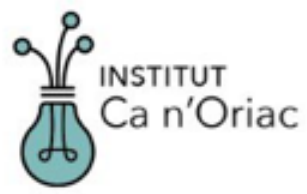

01

Consulta, llegeix i tria la teva càpsula

Les chpsules són propestes dractivitats qae t'ajudaran a dissemyar un itinerari dapenentatge. Clica a cada cipsula i llegeix la seva fitza informativa. Després decideix quines són les tres capsules que met: ragraden. Totes estan pleses fractivitats creatives i que segur tinspiren

Dissenya el teu itinerarl d'aprenentatge

Decideix quina clapsula t'agradaria realitzar en primer lloc, legeix be les indicacions de la capsula (tasques que cal fer o producte final i planifica i dissenya ef teu itinerari d'apeenentatge amb racompanyament de tutot/a i familia. Et suggerim fer un horari que especifiqui la teva dedicacio daria.

Tutories de seguiment I " Help mel " ef teu tutor/a de seguiment. Els dimarts i dimecres de 12 a 13 h Mi haurà el " Hetp met", aqui trobarke les instruccions, una peofessora estara disponible a l'aplicació hangouts per reforgar, si us cal, aquest acomparnyament. sempre podeu contactar amb el professorat a través del comeu electrònic

Fes el lliurament de les propostes de les càpsules L'acompanyament $i \mathrm{t}$ cura seed el centre durant tot el procks Soprenentatge. El likarament i feedback de tot aquest itinerari es realitrarb a traves del teu tutot/a de seguiment segons el canal indicat (CLASspoom.
Is podran realitzar tatories setmanals dilluns i dijous a partir de les $11 \mathrm{~h}$ amb DRNE, correu electrónic)

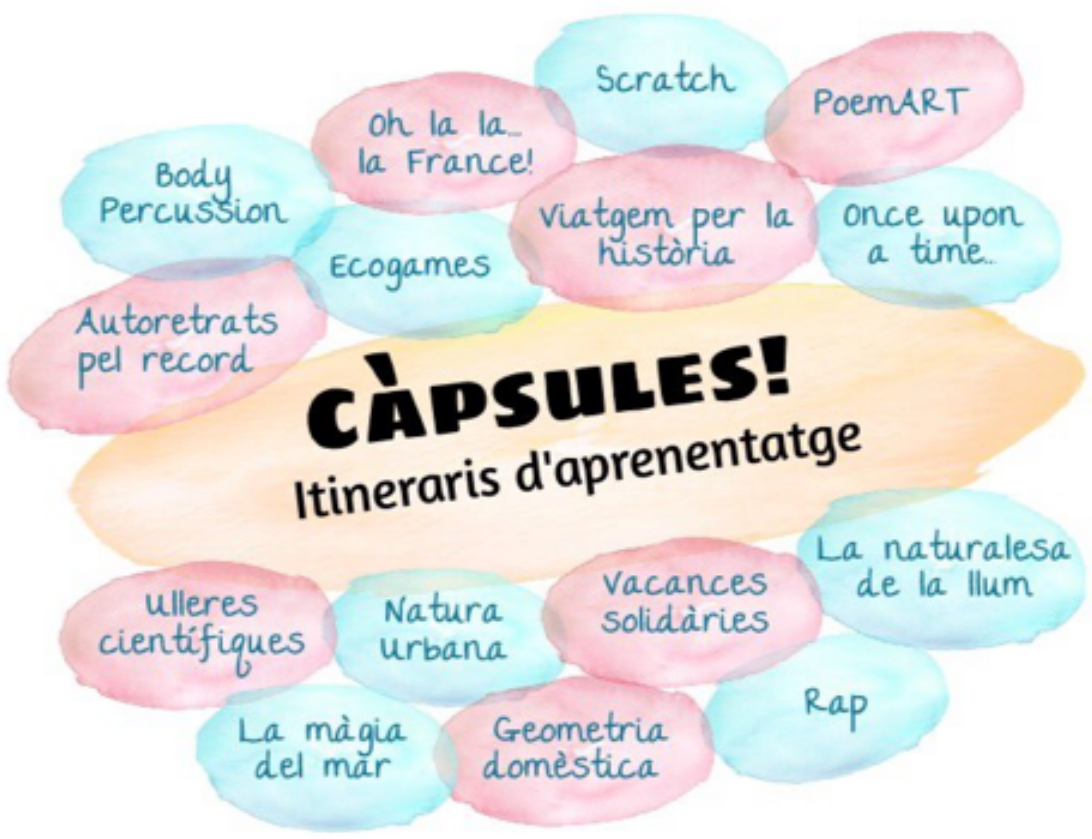

Figura 4. Proyecto ";Cápsulas! Itinerarios de aprendizaje". Fuente: Institut Ca n'Oriac (https://agora.xtec.cat/inscanoriac/). https://doi.org/10.1007/s11858-017-0842-z
3 Se puede consultar el modelo general - plantilla de las cápsulas: https://drive.google.com/file/d/1XZJKxlsise3h9Zgc4Y4w7grijugbDO$\underline{\mathrm{AP} / \mathrm{view}}$ 
un libro viejo o un periódico para arrancar una de sus páginas y reutilizarla con el fin de buscar palabras que aparentemente no han estado destinadas a estar juntas, utilizando técnicas del dadaísmo (ejemplo-modelo Joan Brossa); Así como, 3) traducir el poema de la tarea anterior al ámbito de las cajas mediante la técnica "assemblage" al estilo de Joseph Cornell. Es decir, mediante un proceso artístico a través del cual se obtengan objetos tridimensionales colocando juntos distintos materiales y elementos que no han sido diseñados con fines estéticos, sino que son redescubiertos y combinados por los y las estudiantes para lograr expresar un mensaje o emoción. Las tareas se entregaron a través de las vías habituales utilizadas: Drive, Classroom, email, o Padlet, según la concreción establecida con el tutor o tutora de seguimiento.

El proyecto permite ilustrar, con cierta claridad, las dimensiones o elementos de la personalización educativa descritas anteriormente (ver Figura 1). A continuación, describimos brevemente los mismos con ejemplos del proyecto estudiado. Cabe decir que el proyecto no fue diseñado en base a cada uno de los distintos elementos, pero si bajo una lógica de personalización educativa.

\section{Identidades de los aprendices}

A pesar de que la propuesta no partía explícitamente de las identidades de los y las estudiantes, no se realizaron por ejemplo "artefactos identitarios" como los descritos anteriormente, sí que se tomaron en consideración los intereses y experiencias de los y las participantes en la configuración de sus particulares itinerarios de aprendizaje, así como en la realización de las distintas tareas de aprendizaje. Por ejemplo, en la cápsula "Once upon a time..." los y las estudiantes debían crear una historia (un cuento) eligiendo los personajes, la trama, el idioma (catalán, castellano, inglés, árabe, chino, etc.), así como el formato del mismo. En otro ejemplo de cápsula, "viajemos por la historia", los y las estudiantes identificaron su primer recuerdo de la historia y preguntaron a distintas personas sobre recuerdos históricos, para configurar una línea temporal. Otra actividad, titulada "mi viaje", consistía en elegir un destino turístico para realizar un viaje con la finalidad de conocer una de las primeras civilizaciones que marcaron la historia de la humanidad, como Egipto, la Antigua Grecia, el Imperio Romano o el Imperio Chino. De este modo, los y las estudiantes debían responder a la siguiente tarea: "he elegido dicho país porqué me interesa, me gusta, me llama la atención y quiero aprender sobre...".

\section{Procesos de reflexión}

En todas las actividades propuestas se facilitaron procesos de reflexión mediante "preguntas epistémicas". En el caso de la cápsula "Once upon a time...", por ejemplo, el o la estudiante se preguntaba por sus visiones en relación a ¿qué crees que es un cuento?, ¿te acuerdas de alguna frase de un cuento?, ¿cómo puedes escribir un cuento?, así como las características físicas y psicológicas de los protagonistas del mismo, o el problema que debían abordar. Dichos procesos de reflexión podían estar asociados también a la propia elección. Del mismo modo, en los espacios de tutorías y acompañamiento se ofrecían espacios para reflexionar en relación con los aprendizajes que estaban llevando a cabo, y en la evaluación, se incorporaban sus visiones y perspectivas.

\section{Agencia del aprendiz}

El protagonismo de los y las estudiantes fue una constante en el proyecto. Por ejemplo, en la cápsula "la naturaleza de la luz" ${ }^{\prime 4}$ se propusieron seis tareas basadas en experimentos que los y las estudiantes debían realizar en sus hogares. Encontramos otro ejemplo a través de la realización de un "disco de Newton", anotando las conclusiones a las que se llegaba, así como, documentando el proceso a través de un texto y haciendo fotografías; o bien mediante la creación de una habitación oscura para comprender los estudios de Gerolamo Cardano (1501-1576) relativos al uso de lentes para mejorar la calidad de la imagen. En definitiva, las distintas tareas propuestas, llevadas a cabo por parte de los y las aprendices, permitieron realizar un itinerario a través de la historia de la ciencia con el objetivo de comprender el conocimiento relativo a la naturaleza de la luz. Al igual que en las otras cápsulas, el aprendiz fue el responsable en la organización y desarrollo de las actividades, lo que incluía fijar un horario diario, así como gestionar el tiempo entre la búsqueda de información en internet (no más de una hora seguida) y la realización de los sucesivos experimentos.

\section{Itinerarios de aprendizaje}

Las cápsulas actuaron como puntos de inicio que permitieron diseñar itinerarios conjuntos entre el alumnado, la familia y el tutor o tutora de seguimiento. De acuerdo con las necesidades, vivencias e intereses de los estudiantes, se elegía una determinada cápsula y a partir de la misma se avanzaba bajo la guía y orientación del tutor o tutora. Para ello, cada lunes y jueves a las $11 \mathrm{~h}$ se realizaron videoconferencias con los tutores de seguimiento. Los martes y miércoles de 12 a 13 h estaban destinados a resolver dudas, recibir orientación, así como, acompañar el proceso de aprendizaje (dichas sesiones llevaban por título: "Help me").

\section{Vinculación-conexión múltiple o bimodal}

Las cápsulas propuestas fueron diversas y multidisciplinares, como ilustran los ejemplos descritos anteriormente. Se basaron en un conjunto de materiales y recursos de creación propia diseñados colectivamente por parte del equipo docente. En concreto, la inspiración surgió de la cápsula "poemART", diseñada por los docentes de educación visual y plástica Dimas Fàbregas y Marco Fragoso, la cual sirvió como punto inicial para el diseño de las otras cápsulas. También se incorporó autoría en abierto con materiales y recursos publicados por el Departament d'Educación ${ }^{5}$ de la Generalitat de Catalunya, CESIRE-Àmbit artístic ${ }^{6}$, así como el campus virtual Versembrant ${ }^{7}$. Este último es un espacio gratuito, abierto, de libre acceso, que recoge materiales de los cursos ofrecidos por dicha entidad: cursos de rap, beatmaking, graffiti y breakdance. Versembrant es una cooperativa que se define como escuela popular itinerante con el propósito de fomentar conciencia crítica en adolescentes y jóvenes mediante el arte

\footnotetext{
${ }^{4}$ Ver presentación con las instrucciones: https://view.genial.ly/5e92dc390a072f0d9653fcab/presentation-lanaturalesadelallum

${ }^{5}$ Recursos de la administración (Departamento d'Educació, Generalitat de Catalunya) disponibles en abierto: http://xtec.gencat.cat/ca/recursos/actuacio-extraordinaria-activitats-autoaprenentatge/

${ }^{6}$ Recursos del Centro de Recursos Pedagógicos Específicos de Apoyo a la Innovación y la Investigación Educativa (CESIRE): https://agora. xtec.cat/cesire/categoria/ambits/artistic/

${ }^{7}$ https://campus.versembrant.cat
} 
urbano y el hip hop. La vinculación entre el centro educativo, la administración de la Generalitat de Catalunya y la cooperativa artística y cultural Versembrant, permite ilustrar la noción de vinculación-conexión en el ámbito comunitario que hemos descrito anteriormente.

\section{Acompañamiento}

Finalmente, un elemento nuclear del proyecto fue el rol docente de acompañar, asesorar y guiar, más que instruir. En este sentido, las mismas bases e instrucciones pedagógicas del proyecto explicitaban el respeto y acompañamiento al estado emocional del alumnado, familias y profesorado como propósito subyacente al mismo. Ello se tradujo en tutorías individuales de seguimiento, así como tareas de tutoría de apoyo por parte del conjunto del equipo docente y acompañamiento de pequeños grupos de alumnos y alumnas (entre 10 y 12 estudiantes) a partir de un marco horario de tutorías de seguimiento individual y en pequeños grupos. Lo que supone que el rol tradicional de especialista de materia por parte del equipo docente se transformó en tutor o tutora de apoyo, retroalimentación y seguimiento. Explícitamente, como se ha mencionado anteriormente, se diseñó el espacio de interacción "Help me".

Retos y oportunidades de la personalización educativa. A modo de conclusión

La mejora y transformación educativa es un compromiso consubstancial asociado a las condiciones y condicionantes económicos, sociales y culturales que atraviesan las circunstancias históricas que las instituciones formales no pueden desatender. Ello obliga a establecer dinámicas de colaboración entre sociedad, investigación, formación e innovación en ecosistemas que fomenten la generación de contextos y experiencias de enseñanza y aprendizaje, más allá de los modelos tradicionales basados en la mera transmisión de informaciones y contenidos (Álvarez-Arregui, 2019).

Para ello, deben explorarse -de forma rigurosa y justificada- nuevos diseños híbridos que contemplen la expansión de oportunidades de aprendizaje, tanto a nivel físico-offline, como virtual-online, alrededor de las experiencias y trayectorias de aprendizaje como marco de la actuación educativa (Autores, 2018; Autores, 2019; Autores, 2014). La situación actual, "educación post-pandemia", no hace más que subrayar la necesidad de modelos pedagógicos preparados para los desafíos que emergen en los actuales escenarios, altamente inciertos y complejos ( $\mathrm{Au}$ tores, 2020; Autores, 2020; Pardo y Cobo, 2020).

La personalización educativa, lejos de concebirse como un proceso de individualización del aprendizaje ("customización" educativa), o un proceso de "personificación", persigue aumentar la libertad, control y responsabilidad (agencia) de los estudiantes, incrementando su capacidad para dirigir los procesos de aprendizaje para que éstos puedan traducirse e incorporarse, más fácilmente, en proyectos personales, así como para facilitar aprendizajes con sentido y valor personal (Coll, 2018; Esteban-Guitart e Iglesias, 2020; Jubany, 2012). Lo que conlleva que el rol docente se oriente al encuentro personal (ayuda, orientación, escucha) trascendiendo vínculos meramente instrumentales o funcionales (Pérez Guerrero y Ahedo Ruiz, 2020; Tarabini, 2020).

En este artículo sugerimos un modelo multidimensional de la personalización educativa que considera la necesidad de tener en cuenta, como mínimo, estos seis elementos vinculados a la misma: a) La consideración de las identidades en curso y (co)construcción de los estudiantes como vehículo de movilización e implicación-conexión con los objetivos pedagógicos y curriculares.

b) Los procesos personales y dialógicos-sociales de reflexión.

c) La responsabilidad y capacidad del aprendiz para tomar decisiones en las distintas fases del proceso educativo.

d) El diseño y materialización de itinerarios o trayectorias de aprendizaje.

e) La vinculación-conexión múltiple-bimodal (a nivel micro-psicológico, y a nivel meso-social-comunitario).

f) Así como, el acompañamiento como relación pedagógica basada en el apoyo, seguimiento y retroalimentación.

El proyecto ¡Cápsulas! Itinerarios de aprendizaje nos ha permitido ilustrar parte de estos elementos en un contexto y situación extraordinaria de confinamiento (tercer semestre del curso 20192020). Asociado al mismo, y a las circunstancias de la llamada docencia remota, aparecen algunos retos y desafíos (Trust, 2020).

En primer lugar, la universalidad de la accesibilidad y adaptación de la práctica a estudiantes en riesgo de exclusión social y diversidad funcional. Lo que podríamos considerar bajo la etiqueta de condiciones básicas para la universalidad de la enseñanza híbrida, virtual o remota. Ello requiere contemplar distintas opciones de aprendizaje, pluralización de la enseñanza, para que el aprendiz pueda utilizar aquellas que más se adecuen a sus necesidades, intereses y objetivos de aprendizaje. En segundo lugar, las cuestiones derivadas de la seguridad y respeto a la privacidad de los participantes en la gestión de sus datos (por ejemplo, imágenes). En tercer lugar, la necesidad de desarrollar la competencia crítica digital que permita moverse satisfactoriamente en el "salvaje oeste educativo digital" (Pardo y Cobo, 2020, p. 18). Lo que conlleva anteponer la necesidad pedagógica por delante de la herramienta utilizada (Moravec et al., 2015).

Consideramos que futuras investigaciones son necesarias no solamente para documentar prácticas pedagógicas de cambio e innovación educativa, como la aquí expuesta, sino también para examinar su impacto y contribución en la mejora de aproximaciones como la personalización educativa que se estima relevante en los emergentes escenarios del siglo XXI, más centrados en competencias, redes y alianzas, que en la reducción del proceso educativo a un solo contexto, agente, espacio y tiempo orientado a la transmisión de informaciones y contenidos

\section{Referencias}

Álvarez-Arregui, E. (2019). Evolución de la universidad en la sociedad del aprendizaje y la enseñanza. El valor de las competencias en el desarrollo profesional y personal. Aula Abierta, 48(4), 349-359. https://doi.org/10.17811/rifie.48.4.2019.349-372

Álvarez-Arregui, E. y Arreguit, X. (2019). El futuro de la universidad y la universidad del futuro. Ecosistemas de formación continua para una sociedad de aprendizaje y enseñanza sostenible y responsable. Aula Abierta, 48(4), 447-480. https://doi.org/10.17811/rifie.48.4.2019.447-480

Argüelles, V. (2020). Sociedad, universidad, empresa y talento en momentos de cambio. Aprender y desaparender para volver a emprender. Aula Abierta, 49(3), 332-338. https://doi. org/10.17811/rifie.49.3.2020.325-331

Barron, B. (2006). Interest and self-sustained learning as catalysts of development: A learning ecology perspective. Human Development, 49(4), 193.224. https://doi.org/10.1159/000094368 
Bronfenbrenner, U. (1985). Contextos de crianza del niño. Problemas y prospectiva. Infancia y Aprendizaje, 29, 45-55. https://doi.org/10.1080/02103702.1985.10822058

Bronfenbrenner, U. (1987). La ecología del desarrollo humano. Experimentos en entornos naturales y diseñados. Barcelona: Paidós.

Chen, C. M. (2008). Intelligent web-based learning system with personalized learning path guidance. Computers $\mathcal{E}$ Education, 51(2), 787-814. https://doi.org/10.1016/j.compedu.2007.08.004

Coll, C. (2009). Enseñar y aprender en el siglo XXI: el sentido de los aprendizajes escolares. Dentro de A. Marchesi, J. C. Tedesco y C. Coll (coord.), Reformas educativas y calidad de la educación (pp. 101-112). Madrid: OEI-Santillana.

Coll, C. (2013). El currículo escolar en el marco de la nueva ecología del aprendizaje. Aula de Innovación Educativa, 219, 31-36.

Coll, C. (2014). El sentido del aprendizaje hoy: un reto para la innovación educativa. Aula de Innovación Educativa, 232, 12-17.

Coll, C. (2018). La personalización del aprendizaje escolar, una exigencia de la nueva ecología del aprendizaje. Dosier Graó: La personalización del aprendizaje, 3, 5-11.

Coll, C., Esteban-Guitart, M. e Iglesias, E. (2020). Aprenentatge amb sentit $i$ valor personal. Estratègies, recursos i experiències de personaltizació educativa. Barcelona: Graó.

DiGiacomo, D. K., Van Horne, K., Van Steenis, E. y Penuel, W. (2018). The material and social constitution of interest. Learning, Culture and Social Interaction, 19, 51-60. https://doi.org/10.1016/j.lcsi.2018.04.010

Esteban-Guitart, M. (2016). Funds of identity. Connecting meaningful learning experiences in and out of school. New York; NY: Cambridge University Press.

Esteban-Guitart, M. (2019). Identity in education and education in identities: Connecting curriculum and school practice to students' lives and identities. En P. Hviid y M. Martsin (Eds.), Culture in education and education in culture (pp. 159-175). Switzerland: Springer.

Esteban-Guitart, M. (2020). Covid-19 y educación: Más allá de nuestra zona de desarrollo real. Cultural Praxis. Recuperado de http://culturalpraxis.net/wordpress1/2020/04/11/covid19-y-educacion-mas-alla-de-nuestra-zona-de-desarrollo-real/

Esteban-Guitart, M. y Llopart, M. (2019). La creación y utilización educativa de artefactos identitarios. Profesorado. Revista de currículum y formación del profesorado, 23(2), 321-334. https:// doi.org/10.30827/profesroado.v23i2.9687

Esteban-Guitart, M. y Moll, L. (2014). Funds of identity. A new concept based on Funds of knowledge approach. Culture $\mathcal{E}$ Psychology, 20(1), 31-48. https:/ / doi.org/10.1177/1354067X13515934

Esteban-Guitart, M. y Gee, J. P. (2020). "Inside the head and out in the world." An approach to deep teaching and learning. Multidisciplinary Journal of Educational Research, 10(1), 1-25. https://doi.org/10.447/remie.2020.4868

Esteban-Guitart, M., Serra, J. M. y Vila, I. (2017). Informationalism and informalization of learning in 21st century. A qualitative study on meaningful learning experiences. Social and Education History, 6(1), 1-25. https://doi.org/10.17583/hse.2017.2111

Esteban-Guitart, M., Coll, C. y Penuel, W. (2018). Learning across settings and time in the Digital Age. Digital Education Review, 33, 1-16. https://doi.org/10.1344/der.2018.33.\%25p

Esteban-Guitart, M., DiGiacomo, D., Penuel, W. y Ito, M. (2020). Principios, aplicaciones y retos del aprendizaje conectado. Contextos Educativos, 26, 157-176. https:/ / doi.org/10.18172/con.3966

Gee, J. y Esteban-Guitart, M. (2019). Designing for Deep learning in the context of digital and social media. Comunicar, 58, 9-18. https://doi.org/10.3916/C58-2019-01

González-Patiño, J. (2018). Learn, Teach and Share, Participa- tion in Expanded Educational Communities: Madrid Pikler Seminar as a Practice of Continuing Education between Childhood Professionals. Digital Education Review, 33, 203-216. https://doi. org/10.1344/der.2018.33.203-216

González-Patiño, J. y Esteban-Guitart, M. (2014). Some of the challenges and experiences of formal education in a Mobile-Centric Society. Digital Education Review, 25, 64-86. https:// doi.org/10.1344/der.2014.25.64-86

González-Patiño, J. y Esteban-Guitart, M. (2019). Affinity online: How connection and shared interest fuel learning. Mind, Culture and Activity, 26(4), 383-386. https://doi.org/10.1080/107 49039.2019.1680695

Grant, P. y Basye, D. (2014). Perzonalized learning: A guide to engaging students with technology. Arlington, VA: Internaitonal Society for Technology in Education.

Iglesias, E., González-Patiño, J., Lalueza, J. L. y Esteban-Guitart, M. (2020). Manifiesto en tiempos de pandemia: Por una educación crítica, intergeneracional, sostenible y comunitaria. $R e$ vista Internacional de Educación para la Justicia Social, 9(3), 181-198. https://doi.org/10.15366/riejs2020.9.3.010

Jovés, P., Siqués, C. y Esteban-Guitart, M. (2015). The incorporation of funds of knowledge and funds of identity of students and their families into educational practice. A case study from Catalonia, Spain. Teaching and Teacher Education, 49, 68-77. https://doi.org/10.1016/jtate.2015.03.001

Jubany, J. (2012). Aprendizaje social y personalizado: conectarse para aprender. Barcelona: Editorial UOC.

Martinez, M. (2001). Key design considerations for personalized learning on the web. Educational Technology E Society, 4(1), 26-40.

Minguillón, J., Mor, E., Santanach, F. y Guàrdia, L. (2005). Personalización del proceso de aprendizaje usando learning objects reutilizables. Revista de Educación a Distancia (RED), Núm Monográfico IV, 1-14.

Moravec, J. et al. (2015). Manifiesto 15. Aprendizaje en evolución. Recuperado de https://manifesto15.org/es/

Moronta, I., Rodríguez-Fernández, A. y Fernández-Lasarte, O. (2019). Autoconcepto académico, motivación escolar e implicación escolar del alumnado universitario de República Dominicana y España. Aula Abierta, 48(3), 271-278. https://doi. org $/ 10.17811 /$ rifie.48.3.2019.271-278

Nasir, N. S., de Royston, M. M., Barron, B., Bell, P., Pea, R., Stevens, R. y Goldman, S. (2020). Learning pathways. En N. S. Nasir, C. D. Lee, R. Pea, y M. M. de Royston (Eds.), Handbook of the Cultural Foundations of Learning (pp. 201-218). New York: Routledge.

Oliveira, A. M. C. y Coelho, C. M. M. (2020). Subjective development process as a path to school learning: the classroom as a dialogic relational context. Studies in Psychology, 41(1). https:// doi.org/10.1080/02109395.2019.1710803

Pardo, H. y Cobo, C. (2020). Expandir la universidad más allá de la enseñanza remota de emergencia. Ideas hacia un modelo híbrido post-pandemia. Barcelona: Outliers School.

Penuel, W. y Johnson, R (2016). Review of continued progress. Promising evidence on personalized learning. Boulder, CO: National Education Policy Center.

Pérez Guerrero, J. y Ahedo Ruiz, J. (2020). La educación personalizada según García Hoz. Revista Complutense De Educación, 31(2), 153-161. https:/ / doi.org/10.5209/rced.61992

Perin, D. (2011). Facilitating student learning through contextualization. A review of evidence. Community College Review, 29(3), 268-295.

Reimers, F. M., y Schleicher, A. (2020). Un marco para guiar una respuesta educativa a la pandemia del 2020 del Covid-19. Miraflores, Perú: Enseña Perú. 
Rodríguez-Ruiz, B., Álvarez-Blanco, L., Martínez-González, R. A. y Epstein, J. L. (2019). Presentación del número relación entre centros educativos, familias y entidades comunitarias. Aula Abierta, 48(1), 7-10. https://doi.org/10.17811/rifie.48.1.2019

Solari, M. y Martín, E. (2020). Teachers' professional identity construction: A sociocultural approach to its definition and research. Journal of Constructivist Psychology. https://doi.org/10.10 80/10720537.2020.1852987

Subero, D. y Esteban-Guitart, M. (2020). Más allá del aprendizaje escolar: El rol de la subjetividad en el enfoque de los fondos de identidad. Teoría de la Educación. Revista Interuniversitaria, 32(1), 213-236. https://doi.org/10.14201/teri.20955

Subero, D., Llopart, M., Siqués, C. y Esteban-Guitart, M. (2018). The mediation of teaching and learning processes through identity artefacts. A vygotskian perspective. Oxford Review of Education, 44(2), 156-170. https://doi.org/10.1080/03054985.201 7.1352501

Status, N. L., Falk, J. H., Penuel, W., Dierking, L., Wyld, J. y Bailey, D. (2020). Interested, desinterested, or neutral: Exploring STEM interest profiles and pathways in a low-income urban community. EURASIA Journal of Mathematics, Science and Technology Education, 16(6), em 1853. https://doi.org/10.29333/ ejmste $/ 7927$

Tarabini, A. (2020). ¿Para qué sirve la escuela? Reflexiones sociológicas en tiempos de pandemia global. Revista de Sociología de la Educación-RASE, 13(2), 145-155. https://doi.org/10.7203/ $\underline{\text { RASE.13.2.17135 }}$
Tedesco, J. C. (2001). Educación y hegemonía en el nuevo capitalismo: algunas notas e hipótesis de trabajo. Revista de Educación, $N^{o}$ Extra 1,91-100.

Trust, T. (2020). The 3 biggest remote teaching concerns we nned to solve now. Edsurge. Recuperado de https: / / www.edsurge.com/news/2020-04-02-the-3-biggest-remote-teaching-concerns-we-need-to-solve-now

Vila, I. y Esteban-Guitart, M. (2017). Familia, escuela y comunidad en las sociedades del siglo XXI. Barcelona: Editorial Horsori.

Vygotsky, L. S. (1984). Aprendizaje y desarrollo intelectual en la edad escolar. Infancia y Aprendizaje, 28, 105-116. https://doi.or $\mathrm{g} / 10.1080 / 02103702.1984 .10822045$

Walkington, C. y Hayate, C. A. (2017). Designing learning personalized to students' interests: Balancing rich experiences with mathematical goals. ZDM Mathematics Education, 49(4), 519530. https://doi.org/10.1007/s11858-017-0842-z Vygotsky, L. S. (1984). Aprendizaje y desarrollo intelectual en la edad escolar. Infancia y Aprendizaje, 28, 105-116. https://doi.org/10.1080/021 $\underline{03702.1984 .10822045}$

Walkington, C. y Hayate, C. A. (2017). Designing learning personalized to students' interests: Balancing rich experiences with mathematical goals. ZDM Mathematics Education, 49(4), 519530. https://doi.org/10.1007/s11858-017-0842-z 